\title{
DETERMINAÇÃO DA PRESENÇA DE AFLATOXINAS EM AMOSTRAS DE PAÇOCAS COMERCIALIZADAS EM MONTES CLAROS-MG
}

\author{
Nayara de Cássia Antunes \\ Graduada em Farmácia pelas Faculdades Integradas Pitágoras de Montes Claros - FIPMoc \\ Ronilson Ferreira Freitas \\ Mestrando em Saúde, Sociedade e Ambiente pela Universidade \\ Federal dos Vales do Jequitinhonha e Mucuri - UFVJM \\ ronnypharmacia@gmail.com \\ Vanessa de Andrade Royo \\ Doutora em Ciências Farmacêuticas pela Universidade de São Paulo - USP \\ Docente das Faculdades Integradas Pitágoras de Montes Claros - FIPMoc, \\ da Faculdade de Saúde Ibituruna - FASI \\ e da Universidade Estadual de Montes Claros-Unimontes
}

Recebido em: 03/11/2014

Aprovado em: 24/11/2014

\begin{abstract}
RESUMO
No Brasil, a cultura do amendoim e do milho tem sido foco de estudos, pois esses grãos possuem um consumo considerável pela população, onde os principais consumidores são crianças. Além disso, possui grande influência na exportação, tornando-se necessários programas de monitoramento e controle de aflatoxinas presentes nos grãos, uma vez que essas substâncias são tóxicas, e quando ingeridas podem causar prejuízo à saúde humana e animal. O objetivo deste estudo foi determinar a presença de aflatoxinas em amostras de paçocas comercializadas na cidade de Montes Claros, MG. Foram obtidas em triplicata amostras de paçocas no comércio de Montes Claros, escolhidos de forma aleatória, e conduzidas ao Laboratório de Toxicologia das Faculdades Integradas Pitágoras de Montes Claros - FIPMoc, onde foram identificadas, trituradas, homogeneizadas e analisadas através de Cromatografia de Camada Delgada. Os resultados foram negativos para as amostras analisadas tanto na revelação física (luz UV), quanto na revelação química com ácido sulfúrico. Conclui-se que são importantes testes de controle de qualidade para avaliar se amostras de paçoca de amendoim estão dentro dos padrões estabelecidos pelos órgãos competentes e livres de substâncias tóxicas.
\end{abstract}

Palavras-chave: Aflatoxinas. Micotoxinas. Amendoim.

\section{DETERMINATION OF AFLATOXINS IN AAMPLES PAÇOCAS MARKETED IN MONTES CLAROS- MG}


ANTUNES, N. de C.; FREITAS, R. F.; ROYO, V. de A. Determinação da presença de aflatoxinas em amostras de paçocas comercializadas em Montes Claros-MG

\begin{abstract}
In Brazil, the cultivation of groundnuts and corn has been the focus of studies because these grains have considerable consumption by the population, with children as the main consumers. Besides, it has great influence on exports, making it necessary programs for monitoring and control of aflatoxins in grains, since these substances are toxic when ingested and can cause injury to human and animal health. The aim of this study was to determine the presence of aflatoxins in samples peanut candies marketed in the city of Montes Claros, Brazil. Were obtained in triplicate samples of peanut candies in trade in Montes Claros, chosen at random, and led to the Toxicology Laboratory of Faculdades Integradas Pitágoras Montes Claro - FIPMoc, which were identified, crushed, homogenized and analyzed by Thin Layer Chromatography. The results were negative for the samples both in physical development (UV light), and in chemical development with sulfuric acid. It is concluded that it is important for quality control tests to assess whether samples peanut candy are within the standards established by competent authorities, and free of toxic substances.
\end{abstract}

Keywords: Aflatoxins. Mycotoxins. Peanut.

\title{
1 INTRODUÇÃO
}

Aflatoxicose é caracterizada como uma doença hepática aguda, subaguda ou crônica de humanos e animais, causada por aflatoxinas (AF's), que são metabólitos tóxicos produzidos pelos fungos Aspergillus flavus e A. parasiticus (PIEREZAN et al., 2012). Esses fungos desempenham papel de patógenos oportunistas, causando deterioração de alimentos (PEREIRA; SANTOS, 2011). Os fungos produzem metabólitos tóxicos quando se multiplicam nos alimentos que recebem a denominação genérica de micotoxinas (OLIVEIRA; KOLLER, 2011).

Os fungos são geralmente filamentosos e multicelulares, com crescimento geralmente apical, o que facilita sua proliferação, pois qualquer fragmento hifálico poderá dar origem à outra formação micelial quando colocado em meio apropriado (HERVEY; CHAMPE; FISCHER, 2008). Também são identificados por suas características macro e micromorfológica, que dependendo do tipo de colônia podem ser separados em dois grandes grupos: o das leveduriformes, que apresentam colônias pastosas ou cremosas; e dos bolores, que apresentam colônias filamentosas podendo ser aveludadas, pulverulentas com os mais variados tipos de coloração (CORRÊA, 1998). Portanto, estes são indesejáveis nos alimentos, pela sua capacidade de produzir uma grande variedade de enzimas que provoca a deterioração (GUIMARÃES et al., 2013). 
ANTUNES, N. de C.; FREITAS, R. F.; ROYO, V. de A. Determinação da presença de aflatoxinas em amostras de paçocas comercializadas em Montes Claros-MG

No entanto, a produção de AF's pode variar tanto qualitativa como quantitativamente entre cepas de uma mesma espécie (SILVA et al., 2013).

O Brasil possui condições propícias para o crescimento de todo tipo de fungos produtores de micotoxinas (MAZIERO; BERSOT, 2010). Tais condições como a temperatura, $\mathrm{pH}$ e umidade relativa do ar são favoráveis ao surgimento de diversos fungos e, consequentemente, estes afetam a qualidade do produto (GOULART et al., 2011), podendo provocar três tipos de reações clínicas no ser humano como: as micoses que são infecções ou doenças decorrentes da invasão de um tecido vivo; alergias ou reações de hipersensibilidade; e micotoxicoses que são intoxicações resultantes da ingestão de alimentos contendo metabólitos tóxicos (CORRÊA, 1998). A entrada das AF's no organismo comumente se dá pela via digestiva e sua absorção geralmente causa reações sob forma de hemorragia, ou até mesmo, necroses (MAZIERO; BERSOT, 2010). A presença de micotoxinas em alimentos é um sério problema para a saúde pública e para a qualidade dos alimentos (ROCHA et al., 2008).

Esses fungos são capazes de produzir mais de um tipo de toxina o que dificulta sua identificação no alimento, para um diagnóstico mais eficaz em possíveis contaminações (SCUSSEL, 1998). Outro fator que dificulta a identificação do tipo de toxina que contaminou o alimento é que os fungos, muitas vezes, não são vistos a olho $\mathrm{nu}$, pois o alimento não apresenta nenhuma característica externa que denuncie essa contaminação, ainda que na maioria das situações se mantenham inalteradas suas propriedades organolépticas (BATATINHA et al., 2003). O isolamento de fungos toxigênicos, a partir de alimentos, principalmente grãos estocados em condições recomendadas, não significa obrigatoriamente risco imediato para consumo, isso porque a presença do microrganismo no alimento não é sinônimo que há produção de toxina, pois nem sempre o fungo será capaz ou terá condições apropriadas para a produção das micotoxinas, bem como a eliminação e inativação do fungo não garante a eliminação da toxina que pode continuar presente e ativa no alimento (CONSELHO DE INFORMAÇÕES SOBRE BIOTECNOLOGIA, 2004).

No Brasil, são previstos na legislação os Limites Máximos Tolerados (LMT) de micotoxinas em alimentos segundo a Agência de Vigilância Sanitária (ANVISA, 2011). Porém, em 1976 foi estabelecida a primeira legislação brasileira para aflatoxinas em alimentos, com a Resolução no 34/76 da Comissão Nacional de Normas e Padrões para Alimentos/ Ministério da Saúde (CNNPA/MS), na qual fixa o limite máximo permitido 
ANTUNES, N. de C.; FREITAS, R. F.; ROYO, V. de A. Determinação da presença de aflatoxinas em amostras de paçocas comercializadas em Montes Claros-MG

somando as concentrações das aflatoxinas $\mathrm{B}_{1}$ e $\mathrm{G}_{1}$ em $30 \mu \mathrm{g} / \mathrm{kg}$ (BRASIL, 1977). A ANVISA em 2002, por meio da Resolução RDC no 274/2002 (BRASIL, 2011), internaliza a Resolução MERCOSUL/GMC nº 25/2002 estabelecendo o limite máximo de $20 \mu \mathrm{g} / \mathrm{kg}$ para somatória das aflatoxinas $\mathrm{B}_{1}+\mathrm{B}_{2}+\mathrm{G}_{1}+\mathrm{G}_{2}$. Nessa perspectiva, $\mathrm{o}$ objetivo desse estudo foi determinar a presença de aflatoxinas em amostras de paçocas comercializadas na cidade de Montes Claros, MG.

\section{MATERIAL E MÉTODOS}

Foram utilizadas amostras de paçocas, obtidas em diversos pontos (norte, sul, leste e oeste) da cidade de Montes Claros, norte de Minas Gerais. As amostras foram adquiridas em triplicata totalizando doze amostras, sendo adquiridas ao acaso e sem apresentar indício de deterioração e armazenados de acordo com o recomendado pelo fabricante. As amostras foram conduzidas para o Laboratório de Toxicologia das Faculdades Integradas Pitágoras de Montes Claros - FIPMoc, onde foram identificadas, trituradas, homogeneizadas e analisadas.

Para a detecção das aflatoxinas seguiu-se os procedimentos preconizados por Soares, Rodriguez-Amaya (1989), as análises foram realizadas através da técnica de Cromatografia em Camada Delgada (CCD) de sílica com fluorescência. Para a separação, extração e purificação de aflatoxinas $B_{1}, B_{2}, G_{1}$ e $G_{2}$ foi utilizado o método descrito pelo International Official Methods of Analysis (FIG. 1). Na CCD, utilizou-se um sistema de eluição constituído de tolueno: acetato de etila: ácido fórmico (60:30:10) para separar os compostos, e a observação foi de forma visual, pelo aparecimento de machas fluorescentes, frente à luz UV. A presença de aflatoxinas foi confirmada por revelação química com aplicação de ácido sulfúrico $25 \%$ na cromatoplaca (ROCHA et al., 2008). 
ANTUNES, N. de C.; FREITAS, R. F.; ROYO, V. de A. Determinação da presença de aflatoxinas em amostras de paçocas comercializadas em Montes Claros-MG

Figura 1 - Fluxograma da técnica de separação, extração e purificação para a determinação de aflatoxinas $B_{1}, B_{2}, G_{1}$ e $G_{2}$ em amostras de paçoca

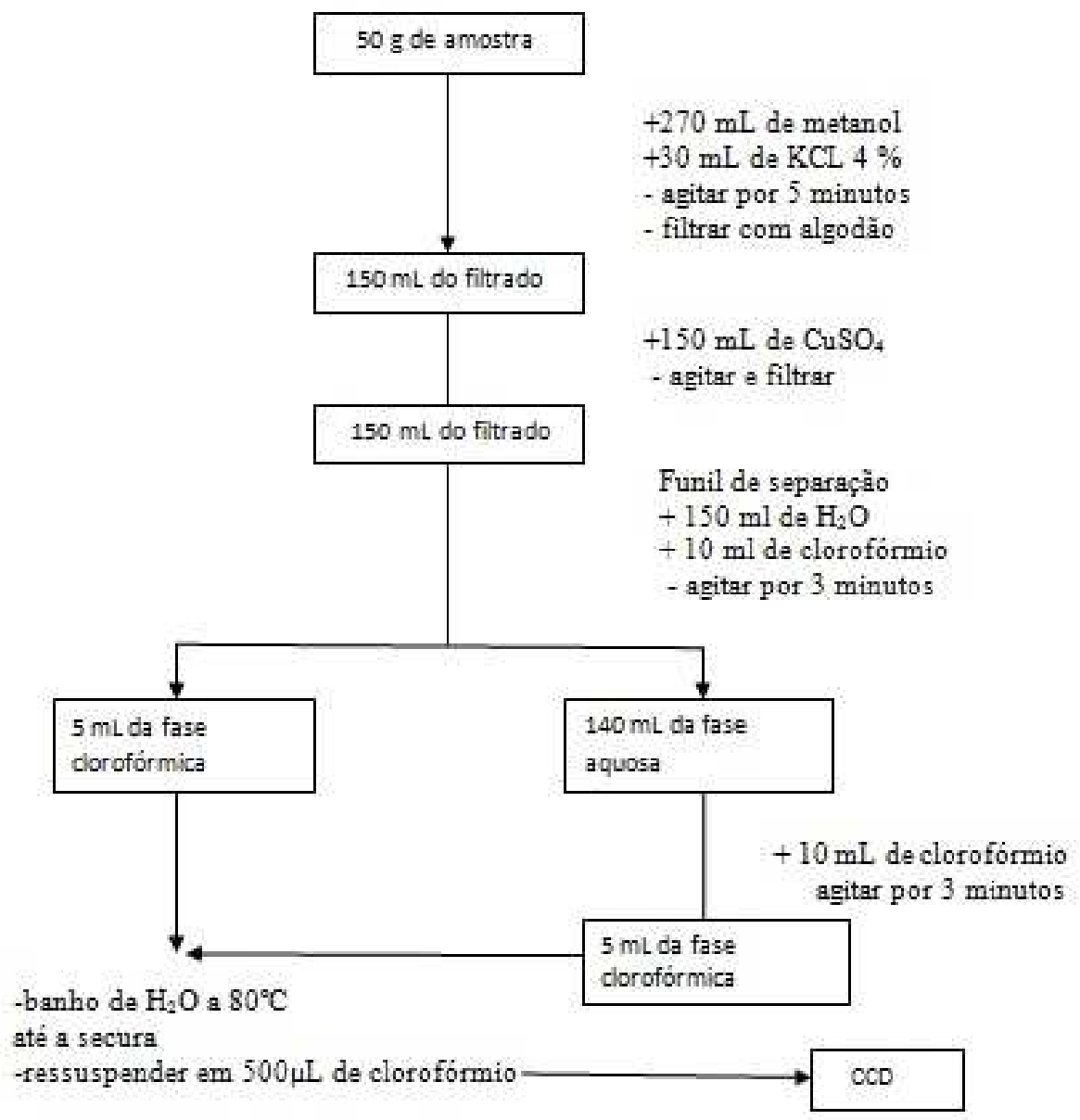

\section{RESULTADOS E DISCUSSÃO}

A incidência de intoxicações humana em decorrência do consumo de alimento contaminado por micotoxinas é uma questão de saúde pública mundial, sendo que essa contaminação dos alimentos pode ocorrer no campo, antes e após a colheita, assim como no transporte e armazenamento do grão; entretanto, estudos revelam que o principal foco de contaminação dos grãos é na colheita e armazenamento (SHUNDO et al., 2010), daí o motivo pelo qual a incidência de aflatoxinas é maior no amendoim, uma vez que o fungo possui maior afinidade pelo produto. Entretanto, seu maior predomínio ocorre quando o amendoim é batido, ensacado e armazenado com umidade elevada ou quando o produto é umedecido depois de seco (GUIMARÃES et al., 2013).

O consumo de amendoim no Brasil chega a 100 mil toneladas de grãos/ano, levando em consideração que há crescimento a cada ano, com concentração da Conexão ci.: r. cient. UNIFOR-MG, Formiga, v. 9, n. 2, p. 38-47, jul./dez. 2014 
ANTUNES, N. de C.; FREITAS, R. F.; ROYO, V. de A. Determinação da presença de aflatoxinas em amostras de paçocas comercializadas em Montes Claros-MG

industrialização na área de confeitos, salgadinhos e doces (SANTOS; LOPES; KOSSEKI, 2001). Esses produtos são largamente consumidos no país, e a existência dessas toxinas em alimentos é extremamente inquietante, levando em consideração que as aflatoxinas possuem a capacidade de se concentrar, isto é, seus níveis vão se acumulando ao longo da cadeia produtiva, uma vez que são moléculas altamente estáveis em diferentes meios bióticos e abióticos, com isso foram desenvolvidos vários programas com intuito de minimizar a incidência de aflatoxinas (QUEZADA, 2000). Em 1989 surgiu o Programa de Melhoria da Qualidade do Amendoim coordenado pelos pesquisadores da Escola Superior de Agricultura "Luiz de Queiroz" da Universidade de São Paulo - ESALQ/USP, caracterizado por um programa de extensão, levando aos produtores instruções sobre práticas recomendadas para manuseio do amendoim (FONSECA, 2013).

Atualmente ainda existem dúvidas a respeito das concentrações máximas de aflatoxinas toleradas em certos alimentos destinados ao consumo humano constituirem risco à saúde pública, mesmo quando encontradas em baixo nível (KENSLER et al., 2011). Assim, é imprescindível a realização de um controle de qualidade pela cadeia produtiva e das ações governamentais para avaliação da qualidade destes produtos, uma vez que são essenciais para evitar riscos à saúde do consumidor e prejuízos econômicos (SILVA et al., 2013).

Em relação às amostras de paçoca de amendoim comercializadas em Montes Claros, apresentou resultado negativo para todas as amostras testadas, tanto na revelação física (luz UV), quanto na revelação química com ácido sulfúrico, entretanto não se pode afirmar que as amostras não estejam contaminadas, uma vez que se existir contaminação com aflatoxina em pequenas concentrações, essas substâncias não são detectadas por CCD (NEVES et al., 2009).

O Brasil tem investido na melhoria da qualidade do amendoim nos últimos anos graças ao desenvolvimento de novas tecnologias (LOURENZANI; LOURENZANI, 2009), em 2002 a Associação Brasileira da Indústria de Chocolate, Cacau, Amendoim, Balas e Derivados - ABICAB determinou a criação de um selo na qual informa ao consumidor que os devidos produtos são seguros para o consumo, pois seguem normas de controle de qualidade estabelecidas pela Food and Agriculture Organization/Organizações das Nações Unidas - FAO/ONU (SHUNDO et al., 2010). Em relação às medidas de prevenção da contaminação por aflatoxinas, o governo 
ANTUNES, N. de C.; FREITAS, R. F.; ROYO, V. de A. Determinação da presença de aflatoxinas em amostras de paçocas comercializadas em Montes Claros-MG

desenvolveu estratégias para reduzir a exposição da mesma, estabelecendo concentrações máximas permitidas em alguns produtos agrícolas destinados ao consumo animal e humano (AMARAL et al., 2006; BENNETT, 2003; IARC, 2002), sendo que a concentração máxima de aflatoxinas tolerada em amendoim e produtos contendo amendoim para o consumo humano é de $20 \mu \mathrm{g} / \mathrm{kg}$, e é considerado o somatório das concentrações dos quatro tipos $\mathrm{B}_{1}, \mathrm{~B}_{2}, \mathrm{G}_{1}$ e $\mathrm{G}_{2}$, (BRASIL, 2011; CALDAS; SILVA; OLIVEIRA, 2002; OLIVEIRA; KOLLER, 2011).

\section{CONCLUSÃO}

Através dos experimentos realizados neste estudo não foi possível determinar a presença de aflatoxinas em amostras de paçocas comercializadas na cidade de Montes Claros, MG. Entretanto, é possível concluir que a realização de controle de qualidade antes e pós-colheita da matéria-prima são indispensáveis para eliminar o crescimento e proliferação de microrganismos capazes de contaminar os alimentos com aflatoxinas.

\section{REFERÊNCIAS}

AMARAL, K. A. S.; NASCIMENTO, G. B.; SEKIYAMA, B. L.; JANEIRO, V.; JUNIOR, M. M. Aflatoxinas em produtos a base de milho comercializados no Brasil e riscos para saúde humana. Ciência e Tecnologia de Alimentos, v. 26, n. 2, p. 336-342, 2006.

ANVISA. Resolução RCD n ${ }^{\circ}$ 07. Dispõe sobre os limites máximos tolerados (LMT) para micotoxinas em alimentos. Agência de Vigilância Sanitária. Diário Oficial da União, 22 de fevereiro de 2011.

AOAC. International Official Methods of Analysis. v. 2, n. 16, p. 2-28, 1999.

BATATINHA, M. J.; SANTOS, M. M.; BOTURA, M. B.; ALMEIDA, G. N.; DOMINGUES, L. F.; KOWALSKI, C. H.; MALLAMANN, C. A. Ocorrência de aflatoxinas em amendoim e seus produtos comercializados no estado da Bahia durante o ano de 2002. Revista do Instituto Adolfo Lutz, v. 62, n. 3, p. 183-187, 2003.

BENNETT, J. W.; KLICH, M. Mycotoxins. Clinical Microbiology Reviews, v. 16, n. 3, p. 497-515, 2003.

BRASIL. Ministério da Saúde. Agência Nacional de Vigilância Sanitária. Resolução RDC $\mathrm{n}^{\circ}$ 7, 18 de fevereiro de 2011. Dispõe sobre limites máximos tolerados (LMT) para micotoxinas em alimentos. Diário Oficial [da] República Federativa do Brasil, Brasília, 9 março 2011. 
ANTUNES, N. de C.; FREITAS, R. F.; ROYO, V. de A. Determinação da presença de aflatoxinas em amostras de paçocas comercializadas em Montes Claros-MG

BRASIL. Ministério da Saúde. Resolução RDC nº 34 de 1976 da CNNPA e ANVISA. Resolve fixar para os alimentos, tolerância de $30 \mathrm{ppb}$ (trinta partes por bilhão) para as aflatoxinas, calculada pela soma dos conteúdos das aflatoxinas B1 e G1. Diário Oficial da União, Brasília, DF, 19 de janeiro de 1977. Seção 1.

CALDAS, E. D.; SILVA, S. C.; OLIVEIRA, J. N. Aflatoxinas e ocratoxina A em alimentos e riscos para a saúde humana. Revista de Saúde Pública, v. 36, n. 3, p. 319$323,2002$.

CONSELHO DE INFORMAÇÕES SOBRE BIOTECNOLOGIA. Ingestão de aflatoxina pode causar câncer. Biotechonology, v. 2, n. 5, p. 01-04, 2004. Disponível em: <http://www.cib.org.br>. Acesso em: 07 jun. 2013.

CORRÊA, B. Fungos toxigênicos: panorama nacional. In: ENCONTRO NACIONAL DE MICOTOXINAS, 9., SIMPÓSIO EM ARMAZENAMENTO QUALITATIVO DE GRÃOS DO MERCOSUL, 1998, Florianópolis. Livro de Resumos. Florianópolis: UFSC, 1998.

FONSECA, H. Micotoxinas on-line. Prevenção e controle de micotoxinas em produtos agrícolas. Práticas usuais e novas dos produtores de amendoim. Disponível em: <http://www.micotoxinas.com.br/Boletim7.htm>. Acesso em: 08 nov. 2013.

GOULART, P. F. P.; ROCHA, J. S.; PIMENTA, C. J.; OLIVEIRA, R. M. E.; CHALFOUN, S. M.; ALVES, A. F. Consumo de aflatoxina B1 e ácidos clorogênicos: um estudo sobre o peso de órgãos e o ganho de peso em ratos Wistar. In: SIMPÓSIO DE PESQUISA DOS CAFÉS DO BRASIL, 7., 2011, Araxá. Anais... Araxá: EMBRAPA, 2011.

GUIMARÃES, K. C. S. S.; FERRO, F. O.; ROCHA, T. J. M.; SILVA, A. C. P.; SILVA, E. C. B. Aflatoxinas: análise por cromatografia em camada delgada e espectrofotometria de amendoins assados coletados em praias da região metropolitana de Maceió-AL. Revista de Biologia Farmácia, v. 9, n. 1, p. 5-13, 2013.

HERVEY, R. A.; CHAMPE, P. C.; FISCHER, B. Microbiologia ilustrada. 2. ed. Porto Alegre: Artmed, 2008.

IARC - International Agency for Research on Cancer. Some traditional herbal medicine, some mycotoxins and styrene. Lyon, 2002. (Monographs on the evaluation of carginogenic risks to humans).

KENSLER, T. W.; ROEBUCK, B. D.; WOGAN, G. N.; GROOPMAN, J. D. Aflatoxin: a 50-year odyssey of mechanistic and translational toxicology. Toxicological Sciences, v. 120, n. 1, p. S28-S48, 2011.

LOURENZANI, W. L.; LOURENZANI, A. E. B. S. Perspectivas do agronegócio brasileiro de amendoim. Information Economics and Policy, v. 39, n. 2, p. 55-68, 2009. 
ANTUNES, N. de C.; FREITAS, R. F.; ROYO, V. de A. Determinação da presença de aflatoxinas em amostras de paçocas comercializadas em Montes Claros-MG

MAZIERO, M. T.; BERSOT, L. S. Micotoxinas em alimentos produzidos no Brasil. Revista Brasileira de Produtos Agroindustriais, v. 12, n. 1, p. 89-99, 2010.

MERCOSUL/GMC (Mercado Comum do Cone Sul/Grupo Mercado Comum). Resolução no 25 de 2002. Regulamento Técnico Mercosul sobre limites máximos de aflatoxinas admissíveis no leite, amendoim e milho. Disponível em:

<http://anvisa.gov.br/legis/resol/mercosul/alimentos/25_02.pdf>. Acesso em: 15 maio 2013.

NEVES, J. A.; SILVA, R. A.; OLIVEIRA, L. R.; BATISTA, R. D. S. R. Determinação da presença de aflatoxinas em castanhas de caju. Publicatio UEPG - Ciências Exatas e da Terra, Agrárias e Engenharias, v. 15, n. 1, p. 39-44, 2009.

OLIVEIRA, L. S. F.; KOLLER, F. F. C. Ocorrência de Aspergillus sp. e aflatoxinas em amostras de amendoim in natura e paçocas. Revista de Ciências Ambientais. v. 5, n. 1, p. 57-68, 2011.

PEREIRA, K. C.; SANTOS, C. F. Micotoxinas e seu potencial carcinogênico. Ensaios e Ciência: Ciências Biológicas, Agrárias e da Saúde. v. 15, n. 4, p. 147-165, 2011.

PIEREZAN, F.; OLIVEIRA-FILHO, J. C.; CARMO, P. M.; AIRES, M. L. R.; SOUZA, T. M.; MALLMANN, C. A.; BARROS, C. S. L. Intoxicação experimental por aflatoxinas em bezerros. Pesquisa Veterinária Brasileira, v. 32, n. 7, p. 607-618, 2012.

QUEZADA, T.; CUÉlLAR, H.; JARAMILLO-JUÁREZ, F.; VALDIVIA, A. G.; REVES, J. L. Effects of aflatoxin B1 on the liver kidney of broiler chickens during development. Comparative Biochemistry and Physiology, v. 125, n. 3, p. 265-272, 2000 .

ROCHA, M. D.; MAIA, P. P.; RODRIGUES, M. A. C.; MARTINS, I. Incidência de aflatoxinas em amostras de amendoim e paçoca comercializadas na cidade de AlfenasMG, Brasil. Revista Brasileira de Toxicologia, v. 21, n.1, p. 15-19, 2008.

SANTOS, C. C. M.; LOPES, M. R. V.; KOSSEKI, S. Y. Ocorrência de aflatoxinas em amendoim e produtos de amendoim comercializados na região de São José do Rio Preto/SP. Revista do Instituto Adolfo Lutz, v. 60, n. 2, p. 153-157, 2001.

SCUSSEL, V. M. Micotoxinas em alimentos. Florianópolis: Insular, 1998.

SHUNDO, L.; NAVAS, A. S., RUVIERI, V.; ALABURDA, J.; LAMARDO, L. C. A.; SABINO, M. Aflatoxinas em amendoim: melhoria da qualidade e programas de controle. Revista do Instituto Adolfo Lutz, v. 69, n. 4, p. 567-570, 2010.

SILVA, R. A.; YAMAMOTO, I. T.; FERREIRA, L. O.; MARQUES, L. R. M. Aflatoxinas em amostras de amendoim cru e derivados. Alimentos e Nutrição (Brazilian Journal of Food and Nutrition), v. 24, n. 1, p. 1-4, 2013. 
ANTUNES, N. de C.; FREITAS, R. F.; ROYO, V. de A. Determinação da presença de aflatoxinas em amostras de paçocas comercializadas em Montes Claros-MG

SOARES, L. M. V.; RODRIGUEZ-AMAYA, D. B. Survey of aflatoxin, ochratoxin A, zearalenone and sterigmatocystin in some Brazilian food by using multitoxin thin-layer chromatographic method. Journal of the Association of Official Analytical Chemists, v. 72 , p. 22-36, 1989. 